\title{
GENERALIZED FRACTIONAL TOTAL COLORINGS OF GRAPHS
}

\author{
Gabriela Karafová And Roman Soták \\ Institute of Mathematics, \\ P. J. S̆afárik University, Jesenná 5, \\ 04001 Košice, Slovakia \\ e-mail: gabriela.karafova@gmail.com \\ roman.sotak@upjs.sk
}

\begin{abstract}
Let $\mathcal{P}$ and $\mathcal{Q}$ be additive and hereditary graph properties and let $r, s$ be integers such that $r \geq s$. Then an $\frac{r}{s}$-fractional $(\mathcal{P}, \mathcal{Q})$-total coloring of a finite graph $G=(V, E)$ is a mapping $f$, which assigns an $s$-element subset of the set $\{1,2, \ldots, r\}$ to each vertex and each edge, moreover, for any color $i$ all vertices of color $i$ induce a subgraph with property $\mathcal{P}$, all edges of color $i$ induce a subgraph with property $\mathcal{Q}$ and vertices and incident edges have been assigned disjoint sets of colors. The minimum ratio of an $\frac{r}{s}$-fractional $(\mathcal{P}, \mathcal{Q})$-total coloring of $G$ is called fractional $(\mathcal{P}, \mathcal{Q})$-total chromatic number $\chi_{f, \mathcal{P}, \mathcal{Q}}^{\prime \prime}(G)=\frac{r}{s}$. We show in this paper that $\chi_{f, \mathcal{P}, \mathcal{Q}}^{\prime \prime}$ of a graph $G$ with $o(V(G))$ vertex orbits and $o(E(G))$ edge orbits can be found as a solution of a linear program with integer coefficients which consists only of $o(V(G))+o(E(G))$ inequalities.
\end{abstract}

Keywords: fractional coloring, total coloring, automorphism group.

2010 Mathematics Subject Classification: 05C15.

\section{REFERENCES}

[1] M. Behzad, Graphs and their chromatic numbers, Ph.D. Thesis, (Michigan State University, 1965).

[2] M. Behzad, The total chromatic number of a graph, a survey, in: Proc. Conf. Oxford, 1969, Combinatorial Mathematics and its Applications, (Academic Press, London, 1971) 1-8.

[3] M. Borowiecki, I. Broere, M. Frick, P. Mihók and G. Semanišin, A survey of hereditary properties of graphs, Discuss. Math. Graph Theory 17 (1997) 5-50. doi:10.7151/dmgt.1037 
[4] M. Borowiecki, A. Kemnitz, M. Marangio and P. Mihók, Generalized total colorings of graphs, Discuss. Math. Graph Theory 31(2011) 209-222.

doi:10.7151/dmgt.1540

[5] M. Borowiecki and P. Mihók, Hereditary properties of graphs, in: V.R. Kulli (Ed.), Advances in Graph Theory (Vishwa International Publication, Gulbarga, 1991) 4168.

[6] A.G. Chetwynd, Total colourings, in: Graphs Colourings, R. Nelson and R.J. Wilson (Eds.), Pitman Research Notes in Mathematics 218 (London, 1990) 65-77.

[7] J.L. Gross and J. Yellen, Graph Theory and Its Applications, (CRC Press, New York 2006) 58-72.

[8] G. Karafová, Generalized fractional total coloring of complete graphs, Discuss. Math. Graph Theory 33 (2013) 665-676. doi:10.7151/dmgt.1697

[9] A. Kemnitz, M. Marangio, P. Mihók, J. Oravcová and R. Soták, Generalized fractional and circular total colorings of graphs, (2010), preprint.

[10] K. Kilakos and B. Reed, Fractionally colouring total graphs, Combinatorica 13 (1993) 435-440. doi:10.1007/BF01303515

[11] E.R. Scheinerman and D.H. Ullman, Fractional Graph Theory (John Wiley and Sons, New York, 1997).

[12] V.G. Vizing, Some unsolved problems in graph theory, Russian Math. Surveys 23 (1968) 125-141.

doi:10.1070/RM1968v023n06ABEH001252

Received 3 April 2014

Revised 3 September 2014

Accepted 3 September 2014 